04

\title{
Разрушение стационарного вихря в упругой среде за счет внешнего энергетического воздействия
}

\author{
(C) В.С. Сухомлинов, ${ }^{1}$ А.С. Мустафраев, ${ }^{2}$ А.А. Страхова, ${ }^{2}$ Н.A. Тимофреев ${ }^{1}$ \\ ${ }^{1}$ Санкт-Петербургский государственный университет, \\ 198504 Санкт-Петербург, Россия \\ ${ }^{2}$ Санкт-Петербургский горный университет, \\ 199106 Санкт-Петербург, Россия \\ e-mail: v_sukhomlinov@mail.ru, alexmustafaev@yandex.ru
}

Поступило в Редакцию 26 июня 2018 г.

В окончательной редакции 31 января 2019 г.

Принято к публикации 28 октября 2019 г.

Рассмотрено решение задачи о динамике структуры бесконечной вихревой трубки в идеальном газе при взрывообразном внешнем подводе энергии вблизи ее оси. Обнаружено, что в пренебрежении вязкостью и теплопроводностью трубка разрушается при сколь угодно малой мощности энерговыделения, при этом время распада вихря уменьшается с ростом суммарной мощности энерговыделения. Построена физическая картина течения, объясняющая обнаруженные закономерности. Получены аналитические соотношения, позволяющие оценить параметры задачи для практического использования взрывчатых веществ при разрушении вихрей большого диаметра. Оценки показывают, что предлагаемый способ практически реализуем.

Ключевые слова: вихревая трубка, внешний энергоподвод, тангенциальная скорость, радиальная скорость, число Маха.

DOI: $10.21883 /$ JTF.2020.04.49079.249-18

\section{Введение}

Существование вихревых образований, в частности в газовой среде, зачастую ухудшает условия функционирования различных систем и механизмов. Примерами могут служить аэродинамическое обтекание в условиях образования турбулентных пограничных слоев [1] (увеличение аэродинамического трения при дозвуковых скоростях, отрыв потока при некоторых режимах обтекания), передача сигнала за счет оптического излучения на большие расстояния в атмосфере [2] и т. п. Особое место в этом ряду занимают вихревые структуры, существование которых в атмосфере сопровождается появлением смерчей, или торнадо. Как известно, зачастую подобные явления приводят к весьма нежелательным последствиям. Указанные обстоятельства, в частности, являются причиной появления большого количества изобретений, касающихся способов разрушения смерчей (торнадо). Известны способы разрушения смерчей (торнадо) за счет внешнего взрывообразного воздействия [3-5]. При этом авторы считают, что энергия взрыва должна быть сравнима с энергией смерча (торнадо) и не проводят количественного анализа временной динамики структуры вихря. В работе [6] предложен способ ослабления смерча за счет искусственной генерации у поверхности земли (в зоне границы воронки смерча) вихрей, имеющих противоположное со смерчем направление вращения. В методе, предложенном в [7], разрушение смерча достигается за счет замедления процессов передачи углового момента вращения из верхней (околооблачной) области торнадо к нижней, находящейся у поверхности земли. Для этого используется доставка в ядро смерча различных химических веществ. Это, по мнению авторов, ведет к замедлению вращения воздушных масс в воронке торнадо.

Отметим, что тема применения внешних воздействий для изменения параметров газовых течений особенно интенсивно развивается в последнее время в связи с попытками оптимизации аэродинамического обтекания нетрадиционными методами - за счет энергетического и силового воздействий на течение [8-10]. Как показано в многочисленных работах, такие методы позволяют существенно улучшить аэродинамические характеристики обтекаемых тел и в ряде случаев дают возможность достигать показателей, реализация которых была невозможной при использовании традиционных способов управления параметрами газовых потоков $[11,12]$.

В работах $[13,14]$ рассматривалось влияние стационарного газового разряда на динамику структуры бесконечной вихревой трубки. Было показано, что если вектор электрического поля в плазме и ось трубки коллинеарны, то вихревая трубка распадается с характерным временем, определяемым параметрами плазмы. Данный эффект объяснялся дополнительным неоднородным энерговыделением в газе, который, в силу рэлеевского механизма, характерен для плазмы самостоятельного газового разряда $[15,16]$.

В настоящей работе рассмотрена пространственновременная динамика структуры вихря при внешнем энергоподводе в газе путем взрывообразного выделения энергии вблизи оси вихря. 


\section{Постановка задачи и получение основных соотношений}

Нетрудно оценить, что при атмосферном давлении, радиусах вихря $r_{0}>10 \mathrm{~m}$ и тангенциальных скоростях $v_{t}$ порядка $10 \mathrm{~m} / \mathrm{s}$ и более, соответствующее рассматриваемому течению число Рейнольдса Re $>10^{7}$. Поэтому рассмотрим стационарную вихревую трубку в изотермическом газе при отсутствии вязкости.

Введем цилиндрическую систему координат, в которой ось $Z$ направлена вдоль оси вихревой трубки. Как известно, структура такой трубки при сделанных допущениях описывается следующими соотношениями [17]:

$$
v_{t}=\omega r \text { при } r<r_{0} ; \quad v_{t}=\frac{\omega r_{0}^{2}}{r} \text { при } r>r_{0} .
$$

При этом давление $\mathrm{P}(\mathrm{r})$ равно

$$
\begin{gathered}
P(r)=P(\infty)+\frac{\rho \omega^{2}}{2}\left(r^{2}-2 r_{0}^{2}\right) \quad \text { при } \quad r<r_{0}, \\
P(r)=P(\infty)-\frac{\rho \omega^{2} r_{0}^{4}}{2 r^{2}} \quad \text { при } r>r_{0},
\end{gathered}
$$

где $\omega$ - частота вращения трубки, $r$ - радиус-вектор, $P(r), \rho$ - давление и плотность газа.

Пусть в момент времени $t=0$ в газе включается источник внешнего энерговыделения объемной мощностью $Q(r, t)$. При сделанных предположениях система уравнений, описывающая дальнейшее поведение трубки, имеет вид [14]:

$$
\begin{gathered}
\frac{\partial P}{\partial t}+v_{r} \frac{\partial P}{\partial r}+\gamma P \frac{1}{r} \frac{\partial\left(r v_{r}\right)}{\partial r}=(\gamma-1) Q(r, t), \\
\frac{\partial v_{r}}{\partial t}+v_{r} \frac{\partial v_{r}}{\partial r}-\frac{v_{t}^{2}}{r}=-\frac{1}{\rho} \frac{\partial P}{\partial r}, \\
\frac{\partial v_{t}}{\partial t}+v_{r} \frac{\partial v_{t}}{\partial r}+\frac{v_{t} v_{r}}{r}=0 \\
\frac{\partial \rho}{\partial t}+v_{r} \frac{\partial \rho}{\partial r}+\rho \frac{1}{r} \frac{\partial\left(r v_{r}\right)}{\partial r}=0
\end{gathered}
$$

где $v_{r}$ - радиальная скорость, $\gamma$ - показатель адиабаты газа.

Перейдем в этой системе уравнений к безразмерным переменным

$$
\begin{gathered}
x=\frac{r}{r_{0}}<1, \quad y=\frac{t a}{r_{0}}, \quad \bar{P}=\frac{P}{P_{0}}, \quad \bar{\rho}=\frac{\rho}{\rho_{0}}, \\
\bar{v}_{x}=\frac{v_{r}}{v_{t}\left(0, r_{0}\right)}, \quad \bar{v}_{y}=\frac{v_{t}}{v_{t}\left(0, r_{0}\right)}, \\
M_{0}=\frac{v_{t}\left(0, r_{0}\right)}{a}, \quad P_{0}=\frac{\rho_{0} a^{2}}{\gamma}, \quad \tilde{Q}=\frac{r_{0}}{a P_{0}} Q, \\
\rho_{0}=\lim _{r \rightarrow \infty} \rho(r), \quad P_{0}=\lim _{r \rightarrow \infty} P(r) .
\end{gathered}
$$

Тогда (3) перепишется в виде

$$
\frac{\partial \bar{P}}{\partial y}+M_{0} \bar{v}_{x} \frac{\partial \bar{P}}{\partial x}+\gamma M_{0} \bar{P} \frac{1}{x} \frac{\partial\left(x \bar{v}_{x}\right)}{\partial x}=(\gamma-1) \bar{Q},
$$

$$
\begin{gathered}
\frac{\partial \bar{v}_{x}}{\partial y}+M_{0} \bar{v}_{x} \frac{\partial \bar{v}_{x}}{\partial x}-M_{0} \frac{\bar{v}_{y}^{2}}{x}=-\frac{1}{\gamma M_{0} \bar{\rho}} \frac{\partial \bar{P}}{\partial x}, \\
\frac{\partial \bar{v}_{y}}{\partial y}+M_{0} \bar{v}_{x} \frac{\partial \bar{v}_{y}}{\partial x}+M_{0} \frac{\bar{v}_{y} \bar{v}_{x}}{x}=0, \\
\frac{\partial \bar{\rho}}{\partial y}+M_{0} \bar{v}_{x} \frac{\partial \bar{\rho}}{\partial x}+M_{0} \bar{\rho} \frac{1}{x} \frac{\partial\left(x \bar{v}_{x}\right)}{\partial x}=0 .
\end{gathered}
$$

Мы рассмотрим тангенциальные скорости вихря, удовлетворяющие неравенству:

$$
\frac{v_{t}^{2}}{a^{2}}=M_{0}^{2} \ll 1,
$$

(где $a-$ скорость звука) поэтому будем оставлять в уравнениях члены, старшие по $M_{0}$. С учетом того что, как легко показать, выполняется

$$
\frac{\Delta \rho}{\rho} \approx \frac{v_{t}^{2}}{a^{2}}
$$

где $\Delta \rho=\rho-\rho_{0}$, после тождественных преобразований из системы (5) получаем уравнение для радиальной скорости $\bar{v}_{x}$ :

$$
\frac{\partial^{2} \bar{v}_{x}}{\partial y^{2}}-\frac{\partial}{\partial x}\left[\frac{1}{x} \frac{\partial}{\partial x}\left(x \bar{v}_{x}\right)\right]=-\frac{(\gamma-1)}{\gamma} \frac{\partial \bar{Q}(x, y)}{\partial x} .
$$

Рассмотрим ситуацию, когда внешний энергоподвод осуществляется в момент безразмерного времени $y=y_{0}$ равномерно по сечению трубки с безразмерным радиусом $\Delta$, т. е. когда

$$
\begin{gathered}
Q(x, y)=\frac{Q_{0} \delta\left(y-y_{0}\right)}{\pi \Delta^{2} r_{0}^{2}} \quad \text { при } \quad x<\Delta, \\
Q(x, y)=0 \quad \text { при } \quad x>\Delta,
\end{gathered}
$$

где $\delta\left(y-y_{0}\right)$ - дельта-функция Дирака, а параметр $Q_{0}$ имеет размерность $\mathrm{W} / \mathrm{m}$.

Применяя преобразование Лапласа и переходя к пределам $\Delta, y_{0} \rightarrow 0$, имеем (см. Приложение 1$):$

$$
\begin{gathered}
\bar{v}_{x}=\frac{\kappa_{0} Q_{0}}{2 \pi} \frac{x}{\left(y^{2}-x^{2}\right)^{1.5}} \quad \text { при } y>x ; \\
\bar{v}_{x}=0 \quad \text { при } y<x,
\end{gathered}
$$

где $\kappa_{0}=\frac{(\gamma-1)}{\gamma M_{0} a r_{0} P_{0}}$. Используя этот результат и третье уравнение системы (5), можно получить (см. Приложение 2): при $x<1$ :

$$
\begin{gathered}
\bar{v}_{y}=x, \text { если } y<x, \\
\bar{v}_{y}=x \exp \left\{-\left(y^{2}-x^{2}\right)\left[1-\frac{1}{\left(1+\beta y \sqrt{\left(y^{2}-x^{2}\right.}\right)^{2}}\right]\right\}, \\
\text { если } y>x,
\end{gathered}
$$

при $x>1$ : 
$\bar{v}_{y}=x^{-1} \exp \left\{-\frac{1}{\beta^{2}}\left[\frac{1}{(\eta+y(\eta))^{2}}-\frac{1}{\eta^{2}}\right]\right\}_{\eta=-\left(y+\frac{1}{\beta \sqrt{y^{2}-x^{2}}}\right)}$,

если $y>x$

где $\beta=\frac{(\gamma-1) Q_{0}}{2 \pi \gamma a r_{0} P_{0}}, y(\eta)-$ решение уравнения (П2.2).

О правильном выборе корней этого уравнения см. Приложение 2 .

При $x>1$ справедливы асимптотические выражения:

$$
\begin{gathered}
\bar{v}_{y}=x^{-1} \exp \left\{-\left(y^{2}-x^{2}\right)\left(1-\frac{1}{\beta^{2} y^{4}}+O\left(\frac{1}{\beta^{3} y^{6}}\right)\right)\right\}_{y \rightarrow \infty}, \\
\text { если } \beta y^{2} \gg 1 \\
\bar{v}_{y}=x^{-1} \exp \left\{-4 \sqrt{2} \beta \varepsilon^{1.5} y x^{1.5}+O\left(\varepsilon^{2.5}\right)\right\}, \\
\text { если } y-x=\varepsilon \ll 1, \quad \varepsilon>0 .
\end{gathered}
$$

Для отношения $\theta$ затраченной энергии на разрушение вихря к первоначальной энергии ядра вихревой трубки можно получить соотношение

$$
\theta=\frac{8 \beta \Delta t a}{(\gamma-1) r_{0} M_{0}^{2}}
$$

где $\Delta t-$ время, за которое выделяется энергия на оси вихря, при этом, согласно соотношению (6), должно выполняться неравенство $\Delta t \ll \frac{r_{0}}{a}$.

Как видно из соотношений $(9),(10)$, после выделения энергии по профилю тангенциальной скорости $\bar{v}_{y}$ от оси вихря бежит волна. Ее скорость $V_{t}(x, y)$ в единицах скорости звука $a$, как нетрудно вычислить, описывается соотношениями

$$
\begin{gathered}
V_{t}(x, y)=\frac{y+f_{1}(x, y) / f(x, y)}{x+f_{2}(x, y) / f(x, y)}, \\
f_{1}(x, y)=\beta \frac{\left(2 y^{2}-x^{2}\right)}{\left(1+\beta y \sqrt{y^{2}-x^{2}}\right)^{3} \sqrt{y^{2}-x^{2}}}, \\
f_{2}(x, y)=\beta \frac{y x}{\left(1+\beta y \sqrt{y^{2}-x^{2}}\right)^{3} \sqrt{y^{2}-x^{2}}}, \\
f(x, y)=1-\frac{1}{\left(1+\beta y \sqrt{y^{2}-x^{2}}\right)^{2}} .
\end{gathered}
$$

\section{Обсуждение полученных результатов}

Отметим, что из формул (9), (10) для тангенциальной скорости следует вывод о том, что вихрь со временем разрушается. Обсудим физические причины этого. При отсутствии внешнего энергоподвода в газе вихревая трубка в отсутствие вязкости стационарна, а роль центростремительной силы играет разница давлений между периферической и центральной областями. В случае выделения энергии на оси трубки происходит рост давления, и центростремительная сила уменьшается. При заданной частоте вращения газа разницы давлений уже не хватает, чтобы удержать газ в равновесии, и в момент времени $t=\frac{r}{a}$ (где $r-$ произвольный радиус внутри вихревой трубки) он начинает смещаться в сторону больших радиусов. Смещение газа в сторону большего радиуса, в свою очередь, в силу законов сохранения ведет к уменьшению частоты вращения, и, таким образом, газ, расширяясь, замедляется. При этом кинетическая энергия вращения переходит в энергию радиального движения газа. Таким образом, по газу со скоростью звука бежит расширяющаяся цилиндрическая волна и при фиксированном радиусе описанные выше процессы начинаются в тот момент, когда волна достигает этого радиуса. Асимптотически при $t \rightarrow \infty$ мы в отсутствие теплопроводности получаем неоднородно нагретый расширяющийся изобарический газ с уменьшающимися радиальной и тангенциальной скоростями.

Интересно отметить, что сам факт распада вихря не зависит от суммарной энергии, выделенной на оси. Таким образом, в отсутствие процессов диссипации энергии (вязкости и теплопроводности) вихрь распадется при сколь угодно малом параметре $Q_{0}$. Более того, как видно из (11), скорость распада вихря на финальной стадии, т. е. при $\beta y^{2} \rightarrow \infty$, вообще не зависит от величины выделенной энергии. В то же время на начальной стадии распада и при относительных временах порядка $y \sim \frac{1}{\beta}$ вихрь распадается тем быстрее, чем больше параметр $Q_{0}$. Это происходит потому, что стационарный вихрь - система неустойчивая по отношению к малым отрицательным возмущениям центростремительной силы. Действительно, при сколь угодно малом ее уменьшении появляется положительная радиальная скорость, происходит увеличение радиуса вращения и, таким образом, увеличение центробежной силы, что ведет к дальнейшему росту радиальной скорости и т.д. Таким образом, система распадается за счет первоначально имеющейся в стационарном состоянии энергии вращательного движения, которая при указанной динамике переходит в энергию радиального движения. Именно поэтому, как можно видеть из полученных формул, скорость распада не зависит также от первоначальной скорости вращения. Классический пример такой системы - груз, вращающийся на струне, которую в некоторый момент времени заменили на растяжимую нить, упругость которой падает с растяжением. Ясно, что груз начнет радиальное движение, одновременно замедляя вращение.

Как следует из соотношения (8), для радиальной скорости при $y<x$ она равна нулю, затем при $y=x$ обращается в бесконечность и при больших значениях $y$ спадает со временем по закону $\sim \frac{1}{y^{3}}$. Разрыв связан тем, что мы предполагали выделение в бесконечно малом объеме конечной мощности $Q_{0}$, т.е. на оси $y$ нас находился источник энергии неограничено большой пространственной плотности мощности. Данное предположение, как нетрудно показать, не меняет основных физических закономерностей рассматриваемого 


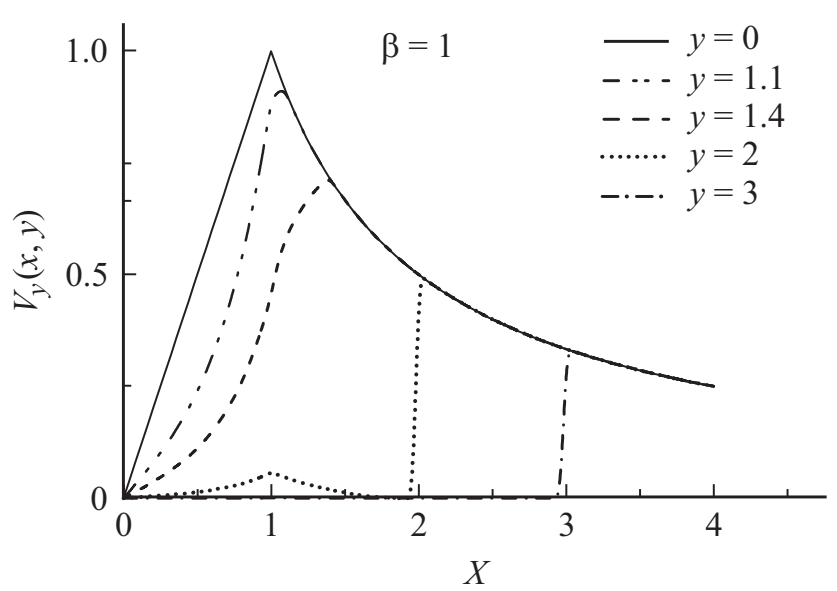

Рис. 1. Зависимость относительной тангенциальной скорости $\bar{v}_{y}$ от безразмерного радиуса $x$ при различных значениях безразмерного времени $y=0,1.1,1.4,2,3, \beta=1$.

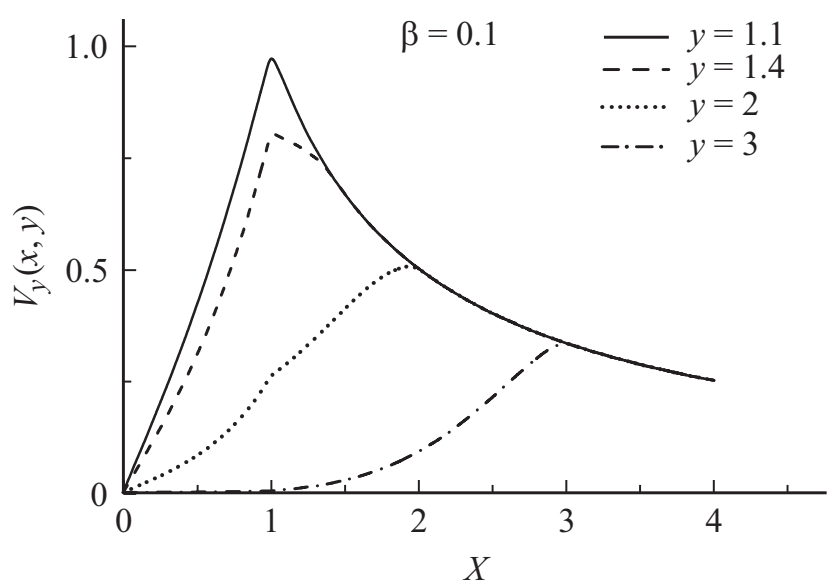

Рис. 2. То же, что и на рис. 1 , но $y=1.1,1.4,2,3, \beta=0.1$.

явления, и при конечной величине $\Delta$, безразмерных временах и радиусах $x \gg \Delta ; y \gg x$ пространственновременная зависимость скорости $\bar{v}_{y}$ будет описываться соотношениями (9), (10).

Оценим безразмерный параметр $\beta$ при атмосферном давлении и температуре порядка $300 \mathrm{~K}$. В этих условиях $\beta \approx 1.3 \cdot 10^{-9} \frac{Q_{0}\left(\frac{\mathrm{W}}{\mathrm{m}}\right)}{r_{0(m)}}$. Если иметь ввиду выделение энергии в результате взрыва, то, полагая, что при взрыве $1 \mathrm{~kg}$ взрывчатого вещества выделяется энергия порядка 4000-6000 $\frac{\mathrm{kJ}}{\mathrm{kg}}$, а детонация происходит со скоростью порядка $10^{4} \mathrm{~m} / \mathrm{s}$, и $1 \mathrm{~kg}$ взрывчатого вещества имеет характерные размеры порядка $10 \mathrm{~cm}$, т. е. детонация $1 \mathrm{~kg}$ вещества происходит за время порядка $10^{-5} \mathrm{~s}$ (при этом характерный размер заданной массы $m$ пропорционален $\left.m^{1 / 3}\right)$, параметра $\beta$ имеем по порядку величины

$$
\beta \approx 10^{3} \frac{m(\mathrm{~kg} / \mathrm{m})^{2 / 3}}{r_{0}(m)},
$$

где $m$ - масса взрывчатого вещества на единицу длины вихревой трубки. Таким образом, при использовании порядка $0.01 \mathrm{~kg} / \mathrm{m}$ вещества и радиусе вихря порядка $50 \mathrm{~m}$ при атмосферном давлении в воздухе $(\gamma=1.4)$ параметр $\beta \approx 1$. Если использовать вещества с меньшей скоростью горения, то параметр $\beta$ уменьшится. При этом, напомним, время распада не зависит от угловой скорости вращения вихревой трубки.

Что касается так называемой энерговыгодности такого способа разрушения вихря, которая определяется формулой (12) ( $\theta<1-$ энерговыгодно и наоборот), то граничное значение параметра $\beta$, при котором $\theta=1$, равно

$$
\beta_{\Gamma}=\frac{(\gamma-1) r_{0} M_{0}^{2}}{8 \Delta t a}
$$

В приведенном выше примере для вихря радиуса $50 \mathrm{~m}$ и начальном числе Маха $M_{0} \approx 0.2$ получаем $\beta_{\text {г }} \approx 6$. Следует, однако, отметить, что при решении поставленной в настоящей работе задачи вопросы энерговыгодности, по-видимому, носят второстепенный характер.

Необходимо также иметь ввиду, что при оценке времен распада больших вихрей (с радиусами порядка $100 \mathrm{~m}$ и более), можно ожидать некоторого влияния

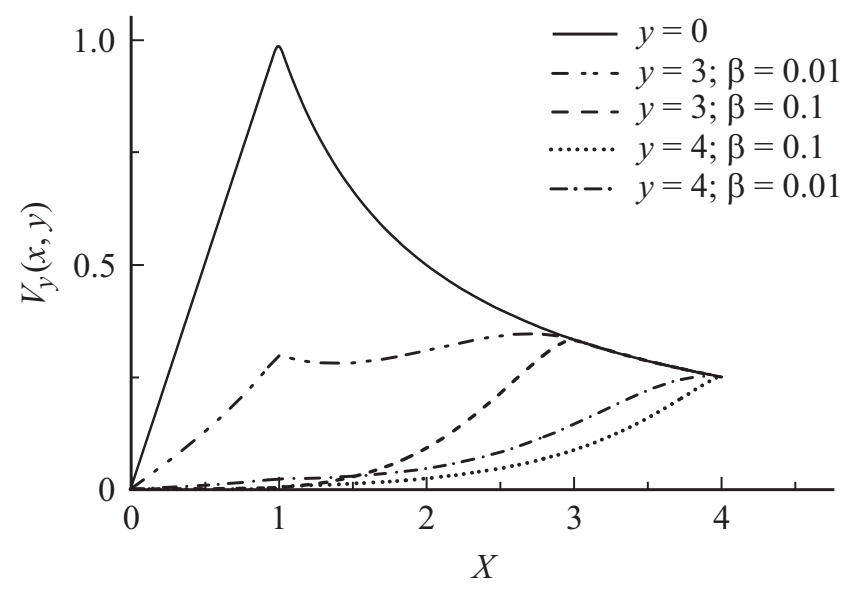

Рис. 3. То же, что и на рис. 1 , но $y=0,2,3, \beta=0.01,0.1$.

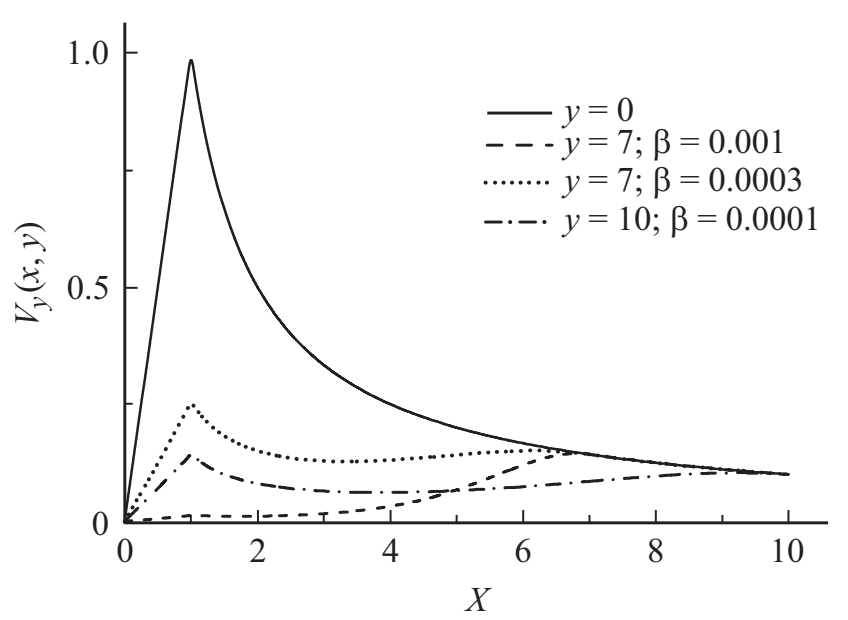

Рис. 4. То же, что и на рис. 1 , но $y=0,7,10, \beta=10^{-3}$, $3 \cdot 10^{-4}, 10^{-4}$. 


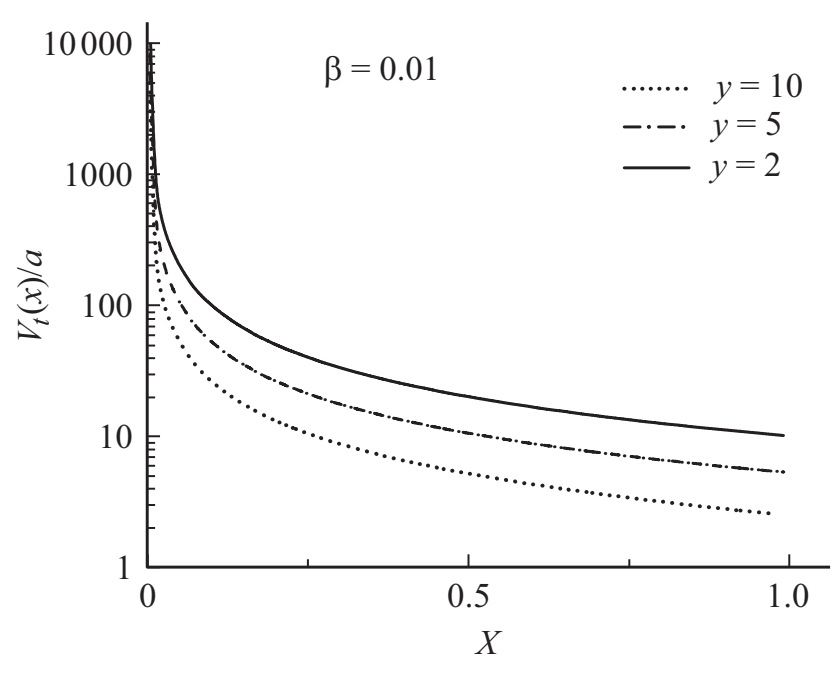

Рис. 5. Пространственные профили скорости перемещения возмущения тангенциальной скорости $V_{t}(x, y)$ при различных безразмерных временах $y=2,5,10$ при $\beta=0.01$.

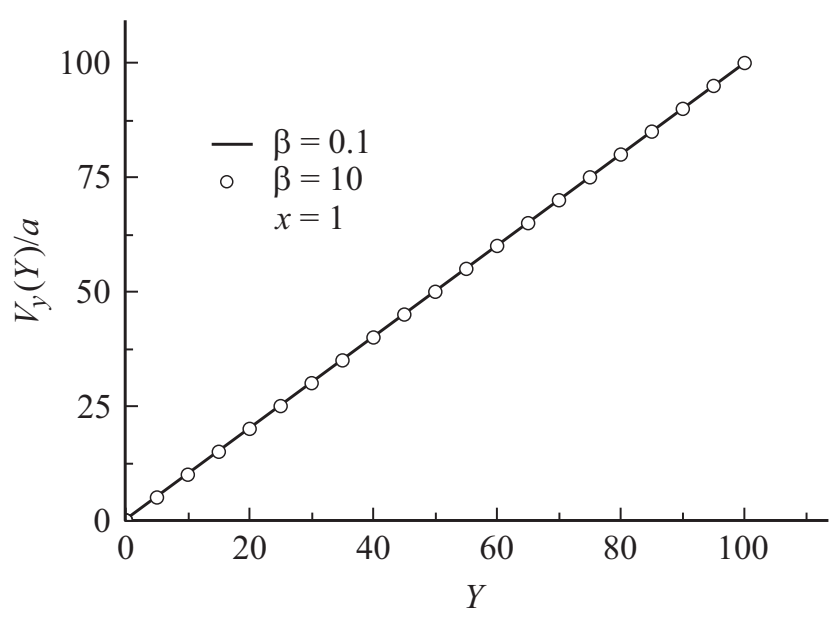

Рис. 6. Временная зависимость величины $V_{t}(x, y)$ при $x=1$ и $\beta=0.1,10$.

вязкости и ослабления бегущей цилиндрической волны, особенно вблизи переднего фронта. Это может ослаблять эффект от энерговыделения в центре вихря и соответственно увеличивать время распада. В большей степени это относится к ситуации, когда мощность энерговыделения мала, а радиус вихря - велик.

Кроме высказанных замечаний по поводу полученных результатов, хочется отметить, что их применимость ограничена также и со стороны больших параметров $\beta$, т.е. при большом энерговыделении в газе. Дело в том, что, как видно из полученных формул, в результате „взрыва“ на оси образуется волна, передний фронт которой распространяется со скоростью порядка звуковой. Это подразумевает, в известной степени, слабое возмущение, поскольку в противном случае образуется ударная волна, скорость которой превосходит скорость звука. Данное ограничение, однако, качественно не ме- няет общую физическую картину происходящих процессов, а лишь сказывается на точности количественного описания динамики структуры вихря.

Указанные выше закономерности иллюстрируют рис. 1-6. На рис. 1,2 приведены расчеты зависимости относительной радиальной скорости от безразмерного времени при различных безразмерных радиусах и параметре $\beta=1,0.1$ соответственно. Видно, что, действительно, по профилю скорости бежит волна, и распад структуры вихря при радиусе гначинается в момент времени $t=\frac{r}{a}$. Безразмерное время распада вихря при этих значениях параметра $\beta$ составляет величину порядка 3-4. На рис. 3, 4 проведено сравнение структуры вихря при разных временах и малых значениях параметра $\beta \simeq 10^{-4}-10^{-1}$. Как и следовало ожидать, поскольку при этих значениях выполняется $\beta y^{2}<1$, скорость распада вихря растет с увеличением параметра $\beta$. При этом при параметре $\beta=10^{-4}$, безразмерное время распада вихря - порядка 10 , что соответствует размерному времени при радиусе вихря порядка $50 \mathrm{~m}$ приблизительно $1.5 \mathrm{~s}$.

На рис. 5 приведены результаты расчетов зависимости скорости возмущения, бегущего по профилю тангенциальной скорости (формула (13)), от безразмерной координаты при различных временах и параметре $\beta=0.01$. Видно, что в центре эта скорость неограниченно возрастает, что является следствием допущения о мгновенном выделении энергии. Далее, по мере роста координаты $x$, скорость падает. Однако при росте времени и фиксированной координате скорость $V_{t}$ растет.

На рис. 6 приведена временная зависимость скорости $V_{t}$ при различных величинах параметра $\beta$ и $x=1$. Видно, что эта скорость линейно растет со временем и скорость этого роста практически не зависит от параметра $\beta$.

\section{Заключение}

В работе решена задача о динамике структуры бесконечной вихревой трубки в идеальном газе при взрывообразном внешнем энергоподводе на ее оси. Интересным, с нашей точки зрения, является тот факт, что в предположении об отсутствии вязкости и теплопроводности вихрь распадается при сколь угодно малой мощности энерговыделения на оси трубки. С физической точки зрения это вызвано тем, что стационарная вихревая трубка неустойчива относительно малых возмущений радиальной скорости, направленных от центра вихря. Ясно, что учет вязкости и теплопроводности газа приведет к существованию некоторой пороговой величины мощности, необходимой для распада вихря.

Картина перестройки течения выглядит следующим образом. По профилю тангенциальной скорости в сторону роста радиуса от оси вихря бежит волна. Ее передний фронт перемещается со скоростью порядка скорости звука, которая слабо зависит от параметра $\beta$. Далее следует область, где возмущение перемещается с различ- 
ной, в зависимости от координаты и времени, скоростью, что приводит к непрерывной перестройке за передним фронтом возмущения всего течения. Этот процесс перестройки существенным образом зависит от параметра $\beta$, и при любом его значении приводит к диссипации вихря. При значительных величинах параметра $\beta$ на профиле тангенциальной скорости образуется волна, подобная ударной с практически вертикальным передним фронтом (рис. 1). Однако в силу использованной системы уравнений и сделанных допущений, скорость распространения переднего фронта возмущения остается близкой к звуковой.

Полученные результаты позволяют оценить параметры задачи для практического использования взрывчатых веществ при разрушении вихрей большого диаметра.

\section{Приложение 1}

Применяя преобразование Лапласа по переменной $y$ к уравнению (6) с учетом (7), имеем для $g(s, x)$

$$
g^{\prime \prime}+\frac{g^{\prime}}{x}-g\left(s^{2}+\frac{1}{x}\right)=-\kappa_{0} \frac{Q_{0}}{\pi \Delta^{2}} \exp \left(-y_{0} s\right) \delta(x-\Delta) .
$$

Уравнение получено с учетом нулевых начальных условий для радиальной скорости и ее производной по времени. Решение этого уравнения для области $x<1$, ограниченное при $x=0$, имеет вид

$$
\begin{aligned}
& g=\kappa_{0} \frac{Q_{0}}{\pi \Delta} \exp \left(-y_{0} s\right) I_{1}(s x) K_{1}(s \Delta) \text { при } \quad x<\Delta ; \\
& g=\kappa_{0} \frac{Q_{0}}{\pi \Delta} \exp \left(-y_{0} s\right) I_{1}(s \Delta) K_{1}(s x) \text { при } \quad x>\Delta,
\end{aligned}
$$

где $I_{1}(x), K_{1}(x)$ - модифицированные функции Бесселя и Ганкеля первого порядка соответственно. Переходя к пределу $\Delta \rightarrow 0$, вычисляя обратное преобразование Лапласа с учетом теоремы о свертке, получим

$$
\begin{gathered}
\bar{v}_{x}=-\kappa_{0} \frac{Q_{0}}{2 \pi} \frac{\partial}{\partial y_{0}} \int_{x}^{\infty} \delta\left(y^{\prime}-y-y_{0}\right) \frac{y^{\prime}}{x \sqrt{y^{\prime 2}-x^{2}}} d y^{\prime} \\
\text { при } \quad y+y_{0}>x \\
\bar{v}_{x}=0 \quad \text { при } y+y_{0}<x .
\end{gathered}
$$

Выполняя вычисления и переходя к пределу $y_{0} \rightarrow 0$, получим формулу (8). Для области $x>1$ решение уравнения (П1.1), ограниченное при $x \rightarrow \infty$, имеет вид

$$
g=C(s) K_{1}(s x) .
$$

Из (П1.2) при $\Delta \rightarrow 0$ из условия непрерывности функции $g(s, x)$ при $x=1$ имеем

$$
C(s)=\kappa_{0} \frac{Q_{0} s}{\pi} \exp \left(-y_{0} s\right)
$$

Из этой формулы следует, что соотношение (8) выполняется при любом значении координаты $x$.

\section{Приложение 2}

Из третьего уравнения системы (5) и соотношения (8) следует, что $\bar{v}_{y}=0$, если $y<x$ при любом значении переменной $x$.

При решении задачи для случая $y>x$ рассмотрим сначала область $x<1$. Вводя в третьем уравнении системы (5) новые независимые переменные

$$
\eta=-\left(y-\frac{1}{\beta \sqrt{y^{2}-x^{2}}}\right),
$$

$y$ вместо переменных $x, y$ и функцию

$$
P(\eta, y)=\ln \left\{\frac{\bar{v}_{y}(x(\eta, y), y)}{x}\right\},
$$

получим уравнение

$$
\frac{\partial P}{\partial y}=\frac{1}{\beta^{2}(\eta+y)^{3}}
$$

решая которое с учетом начальных условий $P(\eta, 0)=0$, имеем

$$
P(\eta, y)=\frac{1}{2 \beta^{2}}\left\{\frac{1}{\eta^{2}}-\frac{1}{(\eta+y)^{2}}\right\},
$$

где $\beta=\frac{M_{0} \kappa_{0} Q_{0}}{2 \pi}$.

Переходя к физическим переменным $y, x$, получаем соотношение (9).

Рассмотрим теперь область $x>1$. Действуя аналогично предыдущему для функции

$$
\bar{P}(\eta, y)=\ln \left\{\bar{v}_{y}(x(\eta, y), y) x\right\},
$$

имеем

$$
\frac{\partial \bar{P}(\eta, y)}{\partial y}=0 .
$$

Граничное условие для этого уравнения - непрерывность функции $\bar{P}(\eta, y)$ при $y=y(\eta)$, где $y(\eta)$ - решение уравнения

$$
\eta=-\left(y+\frac{1}{\beta \sqrt{y^{2}-1}}\right) \text {. }
$$

В результате получаем формулы (10). Отметим, что уравнение (П2.2) имеет два действительных корня $y_{1}(\eta), y_{2}(\eta)$, для которых выполняется асимптотика

$$
\lim _{\eta \rightarrow-\infty} y_{1}(\eta)=1 ; \quad \lim _{\eta \rightarrow-\infty} y_{2}(\eta)=\infty .
$$

При подстановке в (10) необходимо правильно их выбирать. Нетрудно доказать, что при любом значении параметра $\beta>0$ существует такое значение $y=y_{\infty}$, при котором выполняется

$$
{\frac{d \eta}{d y_{y=y_{\infty}}}}=0
$$


и $y_{1}(\eta)=y_{2}(\eta)=y_{\infty}$. Тогда при $y<y_{\infty}$ следует полагать $y(\eta)=y_{1}(\eta)$, а при $y>y_{\infty}-y(\eta)=y_{2}(\eta)$. Легко показать, что имеет место асимптотика

$$
\begin{gathered}
y_{1}(\eta) \cong 1+\frac{1}{2 \beta^{2} \eta^{2}} \quad \text { при } \quad y \rightarrow 1 ; \\
y_{2}(\eta) \cong-\eta+\frac{1}{\beta^{2} \eta} \quad \text { при } \quad y \rightarrow \infty .
\end{gathered}
$$

Таким образом, алгоритм расчета при $x>1$ заключается в следующем. При заданном параметре $\beta$ численно решается уравнение (П2.3) и находится $y_{\infty}$. Если время $y$, при котором необходимо выполнить расчет, меньше этого значения, то при численном решении уравнения (П2.3) в качестве нулевого приближения берется первое из соотношений (П2.4), если больше то второе.

\section{Конфликт интересов}

Авторы заявляют, что у них нет конфликта интересов.

\section{Список литературы}

[1] Clauser F.H. // Advan. Appl. Mechan. 1956. Vol. 4. P. 1-51.

[2] Khmelevtsov S.S. // Appl. Opt. 1973. Vol. 12. N 10. P. 24212433.

[3] Feldman B. Method of and system for destabilization and destruction of dangerous atmospheric phenomena mainly tornado // US 20020088364 A1. 2002.

[4] Nelson R.H. Tornado disarming network // US 20100001118 A1. 2010.

[5] Gravina M.B. Thermal Energy Radiance Expander // US 20100276533 A. 2010.

[6] Henry $\mathrm{Yi}$, Jane $\mathrm{Yi}$. Tornadoes dynamic teardown system (TDTS) // US 20040149156 A1. 2004.

[7] Bissell A.M. Methods and apparatus for destabilizing tornadoes // WO 2013155051 A1.2013.

[8] Simmons G.A., Nelson G.L. Overview of the NASA MARIAH project and summary of technical results. AIAA 1998-2752 Albuquerque, NM June 1998.

[9] Kolosov V.Yu., Sepman V.Yu., Sukhomlinov V.S., Tolmachev Yu.A., Kuranov A.L., Kuchinsky V.V., Sheverev V.A., Otugen M.V. „Effect of acoustic dispersion on the propagation of a shock wave in a glow discharge“, Proceedings of 9th International Space Planes and Hypersonic Systems and Technologies Conference, Norfolk, VA, Nov. 1-5. 1999. AIAA. P. 1999-4882.

[10] Голятин В.Ю., Кучинский В.В., Сухомлинов В.С. // ЖТФ. 2005. Т. 50. Вып. 5. С. 52-59.

[11] Gurijanov E.P., Harsha P.T. AJAX - new directions in hypersonic technology, AIAA 1996-4609, Norfolk VA, November 1996.

[12] Герасимов Н.А., Сухомлинов В.С. // ЖТФ. 2010. Т. 55. Вып. 11. С. 6-10.

[13] Sheverev V.A., Otugen M.V., Sukhomlinov V.S. // Phys. Fluid. 2005. Vol. 17. N 5. P. 1-4.

[14] Сухомлинов В.С., Мустафаев А.С. // ЖТФ. 2016. Т. 61. Вып. 9. С. 48-54.
[15] Gerasimov N., Sheverev V.A., Sukhomlinov V.S. // J. Appl. Phys. 2009. Vol. 104. N 4. P. 043301.

[16] Gerasimov N.A., Kuchinskiy V.V., Sukhomlinov V.S., Sukhomlinov S.V. // Tech. Phys. 2007. Vol. 52. N 7. P. 831-838.

[17] Бондарев Е.Н., Дубасов В.Т., Рыжсов Ю.А. // Аэродинамика. М.: Машиностроение, 1993. С. 90-91. 